\title{
Reproducción y crecimiento del pargo mancha Lutjanus guttatus (Pisces: Lutjanidae) en el Golfo de Nicoya, Costa Rica
}

\author{
Rosa L. Soto Rojas, Fernando Mejía-Arana, J. A. Palacios \& Kazuhito Hiramatsu \\ Estación de Biología Marina, Escuela de Ciencias Biológicas, Universidad Nacional, Puntarenas, Costa Rica, Tel. \\ (506)-2661-26-70, Fax (506) 2661-36-35. rsoto@una.ac.cr; jpalacio@una.ac.cr; kazuhiramartsu@gmail.com
}

\author{
Recibido 08-IV-2008. Corregido 22-VI-2008. Aceptado 28-VII-2008.
}

\begin{abstract}
Reproduction and growth of the fish Lutjanus guttatus (Pisces: Lutjanidae) in the Gulf of Nicoya, Costa Rica. The fish Lutjanus guttatus is important in the fisheries of Golfo de Nicoya, Costa Rica where they are captured with varied gear mainly by the artisanal fleet. We measured specimens from the commercial catch of 2002-2006. Gonadal state was determined macroscopically and age with otoliths The total length-total weight relationship was $\mathrm{y}=0.0236 \mathrm{x}^{2.8153}$ and total length-eviscerated weight $\mathrm{y}=0.0216 \mathrm{x}^{2.8129}$. Simplified relationships were $y=0.0173 \times 3$ and $y=0.0162 \times 3$. There is year-round reproduction with peaks in March (dry season) and September (rainy season). Male-female sexual ratio was 1:1. The age-length key for the gulf shows availability until the 6 years of age. The von Bertalanffy growth curve is $\mathrm{L}(\mathrm{t})=65,9\left(1-\mathrm{e}^{-0,13(t+2,66)}\right)$. Rev. Biol. Trop. 57 (1-2): 125-131. Epub 2009 June 30.
\end{abstract}

Key words: Lutjanus guttatus, Lutjanidae, spotted snapper, reproduction, growth, age, morphometric relationships, Golfo de Nicoya.

Como parte de las especies de un fuerte aprovechamiento dentro de los recursos marino-costeros de Costa Rica y que forman parte de las pesquerías en el Golfo de Nicoya está la familia Lutjanidae; conformada por diez especies (nueve especies del género Lutjanus y Hoplopagrus guntheri) en el Pacífico de Centroamérica Meridional (Bussing 1994, Anderson y Newman 1996, Márcano et al. 2002, Robertson y Allen 2002). En este grupo de lutjánidos el pargo mancha (Lutjanus guttatus, Steindachner 1869) es importante comercial y ecológicamente, sus especímenes viven en pequeños grupos o solitarios, pocas veces conformando grandes cardúmenes, se localizan en zonas costeras hasta $30 \mathrm{~m}$ de profundidad, son carnívoros y se alimentan principalmente de peces e invertebrados (Fischer et al. 1995).

En 1998 Vargas, caracterizó al pargo mancha como una especie altamente capturada por la flota artesanal en el Golfo de Nicoya, principalmente en la parte externa, siendo la especie que sostiene las pesquerías en esta zona del Golfo. Rojas (1997) la considera de alta importancia en las capturas nacionales, con producciones mensuales registradas superiores a 3 millones de dólares (setiembre del 2003).

Araya et al. (2007) establecen el caladero de captura del stock de pargo mancha en las zonas 3 y 4 del Golfo de Nicoya (según legislación actual), sin que las capturas presenten una tendencia clara pero con variaciones entre 1994-2005 de 118 a 299 toneladas anuales. Así mismo, se sienta que los artes de pesca utilizados en la captura del pargo mancha son la línea de fondo y el trasmallo, faenados principalmente por la flota artesanal del Golfo de Nicoya (Araya et al. 2007).

El objetivo de esta investigación es conocer las principales características morfométricas, 
reproductivas y de crecimiento de la población de pargo mancha presente en el Golfo de Nicoya.

\section{MATERIALES Y MÉTODOS}

Se realizaron cuatro campañas de muestreo entre el 2002 y el 2006, incluyéndose un año completo de muestreos entre diciembre del 2002 a noviembre del 2003 y algunos meses en los otros años. A cada espécimen se le midieron las siguientes variables morfométricas: longitud total $(\mathrm{Lt})$, peso total $(\mathrm{Pt})$ y peso eviscerado $(\mathrm{Pd})$. Se establecieron las relaciones entre la longitud total-peso total y la longitud totalpeso eviscerado, para precisar su crecimiento haciendo uso de la siguiente fórmula: $\mathrm{P}=\mathrm{aLt} \mathrm{t}^{\mathrm{b}}$ (Sparre y Venema 1995).

Para determinar el periodo de reproducción se hizo el análisis de las gónadas de las hembras a través de la observación macroscópica, utilizando la escala de madurez gonadal propuesta por Pollar (1972), y posteriormente se aplicó el Índice Gonadosomático (IGS) según Tanaka (1985).

Para la determinación de la edad los otolitos sagitta fueron previamente embebidos en resina (Quetol 812) y luego se hicieron cortes transversales con un micrótomo de partes duras, luego se pulieron hasta permitir la observación apropiada con un microscopio óptico (Kimura 2003; Shimose y Tachihara 2005).

Los anillos anuales se establecieron a través de la observación de los cambios de dirección del crecimiento; para establecer la fecha de nacimiento del espécimen se utilizó la fecha de captura, los anillos anuales y el incremento del borde final del último anillo.

También, se calcularon los parámetros de crecimiento $\left(\mathrm{L}_{\infty}, \mathrm{K}\right.$ y t $\left.\mathrm{t}_{0}\right)$ de acuerdo a la metodología propuesta por Sparre y Venema (1995), para luego ser aplicados a la curva de crecimiento de von Bertalanffy, $\mathrm{L}(\mathrm{t})=\mathrm{L}_{\infty}$ * $\left[1-\exp \left(-\mathrm{K}^{*}\left(\mathrm{t}-\mathrm{t}_{0}\right)\right)\right]$.

\section{RESULTADOS}

Usando líneas de tendencia potencial $\left(\mathrm{y}=\mathrm{cx}^{\mathrm{b}}\right)$ en Excel, se pudieron determinar las ecuaciones de relación corporal para la especie, como se observa en las Figuras 1 y 2. Así, la relación Lt-Pt se presentó como $\mathrm{y}=0,019 \mathrm{x}^{2.867}$, $\mathrm{y}$ la relación Lt-Pd como $\mathrm{y}=0,017 \mathrm{x}^{2.872}$; estos resultados muestran que esta especie presenta un crecimiento alométrico negativo (King 1995). Así mismo, al realizar la correspondiente simplificación $(b=3)$ de las relaciones morfométricas, se obtuvieron las siguientes ecuaciones: $\mathrm{y}=0,0173 \mathrm{x}^{3}$ para la relación longitud total-peso total y $\mathrm{y}=0,0162 \mathrm{x}^{3}$ para la relación longitud total-peso eviscerado.

En la Figura 3 se puede observar que el periodo de reproducción del pargo mancha para el Golfo de Nicoya se presenta durante todo el año, con dos máximos importantes: en marzo y septiembre, coincidiendo con el final de la época seca, y durante la época lluviosa; con los correspondientes valores del índice gonadosomático (IGS) para marzo de 4.4 y para setiembre de 4.1. Además se calculó la relación hembra-macho siendo el resultado de 1:1.

En la determinación de edad (Cuadro 1) se puede observar que, cuando las tallas están

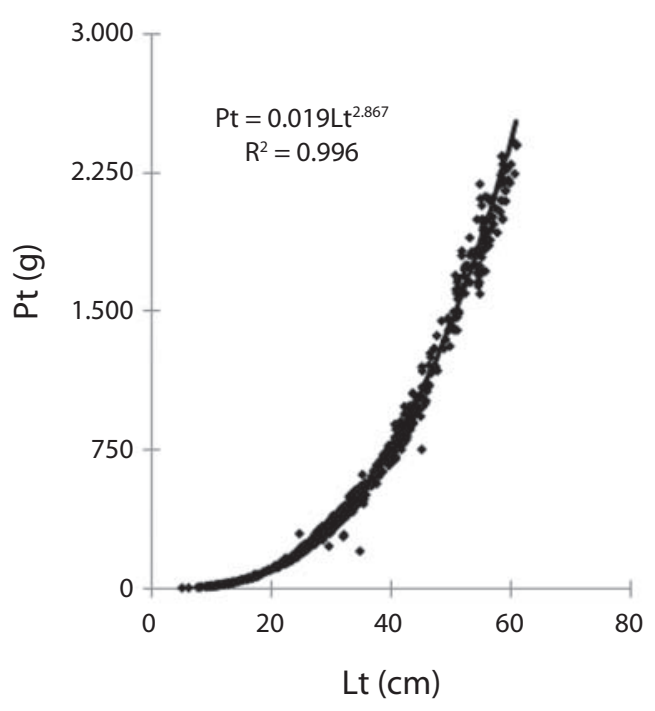

Fig. 1. Relación Lt-Pt del pargo mancha en el Golfo de Nicoya, Costa Rica.

Fig. 1. TL-BW relation for pargo mancha in the Gulf of Nicoya, Costa Rica. 


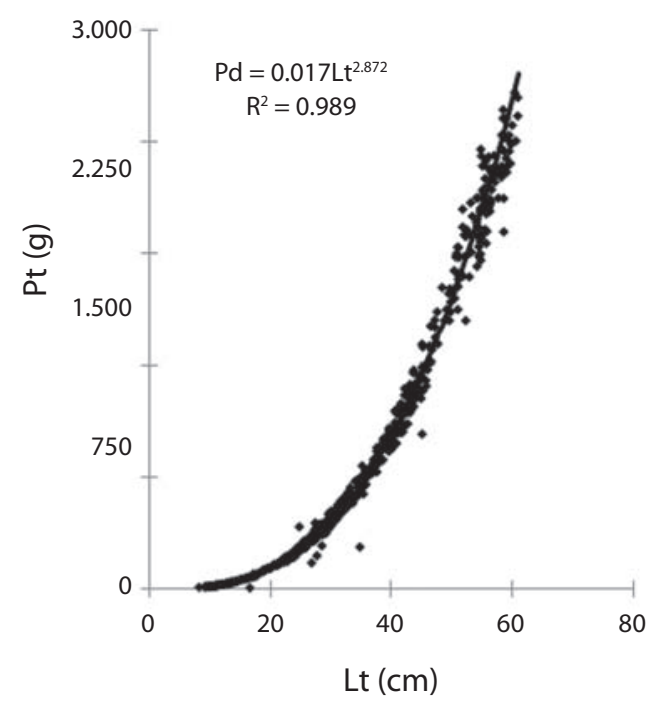

Fig. 2. Relación Lt-Pd del pargo mancha en el Golfo de Nicoya, Costa Rica.

Fig. 2. TL-GW relation for pargo mancha in the Gulf of Nicoya, Costa Rica.

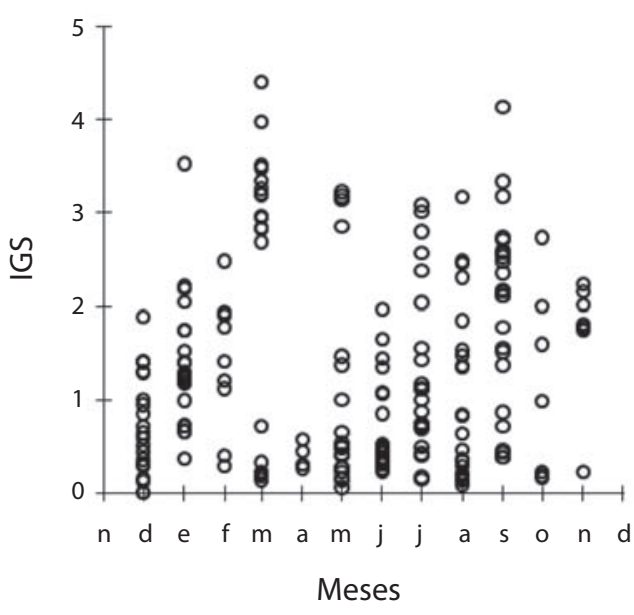

Fig. 3. Índice gonadosomático del pargo mancha en el Golfo de Nicoya.

Fig. 3. Gonadosomatic index (GSI) for pargo mancha in the Gulf of Nicoya, Costa Rica.

CUADRO 1

Clave talla-edad (porcentajes de especimenes) para pargo mancha en el Golfo de Nicoya, Costa Rica

TABLE 1

Age-length key (specimens' percentage) for pargo mancha in the Gulf of Nicoya, Costa Rica

$$
\begin{aligned}
& \text { Lt }(\mathrm{cm}) \\
& 18-26 \\
& 27-36 \\
& 37-46 \\
& 47-56 \\
& 57-60
\end{aligned}
$$

entre los 18 y $26 \mathrm{~cm}$ el mayor porcentaje de especímenes presentan edades entre 1 y 2 años $(82 \%)$, para los de 27 a $36 \mathrm{~cm}$ de talla el mayor porcentaje tienen edades desde 1 a 3 años $(69 \%)$, producto de traslapes entre clases, situación normal en la naturaleza; mientras, los especímenes de tallas entre los 37 y $46 \mathrm{~cm}$ presentan edades entre 3 y 4 años principalmente

\section{Edad (años)}

3

4

5

6

7

26

29

14

27

$\begin{array}{ccc}9 & 5 & \\ 15 & 9 & 3 \\ 14 & 7 & \end{array}$

11

11

28

(56\%). En el caso de los especímenes con tallas de 47 a $56 \mathrm{~cm}$ poseen 5 años de edad, y los especímenes con tallas de $57 \mathrm{~cm}$ o superiores presentan más de seis años de edad.

Los parámetros poblacionales obtenidos son: $\mathrm{L}_{\infty}=65,9 \mathrm{~cm}, \mathrm{~K}=0,13 \mathrm{y} \mathrm{t}_{0}=-2,66$, los cuales al ser aplicados en la curva de crecimiento de von Bertalanffy brindan la Figura 4. 


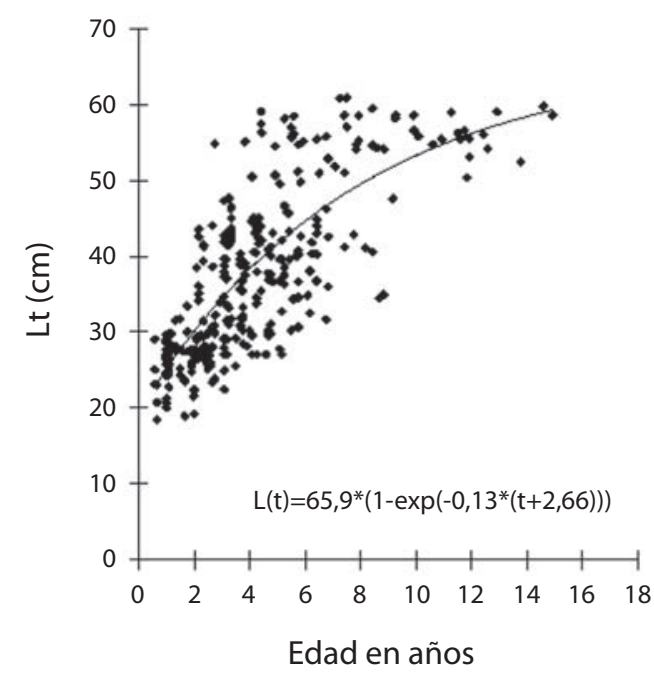

Fig. 4. Anillos de crecimiento (flecha negra) en otolitos sagitta de pargo manch: a. anillos anuales, b. anillos diarios.

Fig. 4. Growth rings (black arrow) in pargo mancha sagitta otolith: a. annual rings, b. daily rings.

\section{DISCUSIÓN}

La alometría que presentan los datos demuestra un crecimiento volumétrico no proporcional (sin una fuerte tendencia cúbica), lo que indica que las relaciones talla-peso encontradas son características propias de la especie, como lo confirma Froese, R. y D. Pauly (2007). Sin embargo, la escasa diferencia entre las pendientes de las relaciones Lt-Pt y Lt-Pd posiblemente sea debido a que la mayor proporción de los especímenes analizados no presentaron contenido estomacal, lo cual pudo haber influido en que el peso total y el desvicerado fueran similares. Por otro lado Vargas (1998) establece que la relación longitud total-peso total no presenta diferencia significativa entre sexos.

La proporción sexual (macho-hembra) encontrada coincide con la reportada por Vargas (1998) y Arellano-Martínez et al. (2001), pero a su vez difiere con lo reportado por Rojas (1997), ya que este determina para el Golfo de Nicoya una proporción sexual de 1.3 machos por hembra. Tal como indica Rojas (1997), esta diferencia puede atribuirse a un comportamiento gregario en función de distribución y demanda de alimento. En conformidad con Claro (1994), se establece que variaciones mensuales en las proporciones sexuales tienden a equilibrase durante la época de desove, por lo cual las variaciones observadas podrían estar estrechamente relacionadas con la época del año, la disponibilidad de recursos y los niveles de madurez reproductiva en el momento de la captura de los especímenes.

Esta especie se reproduce durante todo el año, con dos máximos importantes en marzo y setiembre; presentando una pequeña diferencia con lo reportado por Rojas (1997). Precisamente es Rojas (1997) quien indica que los picos de desoves son en abril y octubre, posiblemente producto del comportamiento mismo de la especie. También, los presentes resultados coinciden con los reportados por ArellanoMartínez et al. (2001) para el pargo mancha en las costa del Estado de Guerrero (México), donde los máximos reproductivos se dan de marzo a abril y de agosto a noviembre. Con respecto a la talla de primera madurez según Rojas (1997), se presenta entre 31,7 y 34,3 cm para la población del Golfo de Nicoya.

La clave talla-edad permite observar un traslape de edades propio de las cohortes de la población a lo largo del tiempo (Sparre y Venema 1995). Por ello la necesidad de establecer un grupo de edad 6 como máximo para el uso de esta clave cuando se utilizan otolitos; $\mathrm{y}$, por tanto, se deben agrupar los especímenes de mayor edad en un grupo $6+$, lo cual permite una adecuada interpretación de las lecturas de otolitos y correspondiente aplicabilidad de la información.

De acuerdo a lo reportado por Vargas (1998), los parámetros de la curva de crecimiento para pargo mancha no difieren mucho de los encontrados en la presente investigación. Incluso, la familia Lutjanidae presenta altas longevidades y crecimiento lento (Vargas 1998, Kamukuru et al. 2005). Por ello, cuando se comparan los parámetros poblacionales de L. guttatus con lo reportado por otros investigadores (Cuadro 2), es posible dilucidar que las divergencias 
CUADRO 2

Valores comparativos de los parámetros de la curva de crecimiento para pargo mancha

TABLE 2

Comparative values of growth curve parameters for pargo mancha

$\begin{array}{lccc}\text { Autor } & \mathrm{L} \infty(\mathrm{cm}) & \mathrm{K} & \mathrm{t}_{0}(\text { años) } \\ \text { Vargas (1998) } & 67 & 0,3 & \\ \text { Fishbase (2007) } & 64,2 & 0,19 & -0,72 \\ \text { Cruz y Chaves (1993) } & 64 & 0,19 & \\ \text { Siefke (1995) } & 83 & 0,15 & \\ \text { Fisher } \text { et al. (1995) } & 80 & & \\ \text { Rojas (1997) } & 60 & & \\ \text { Andrade (2003) } & 66,4 & 0,13 & 0,03 \\ \text { Este Trabajo (2007) } & 65,9 & 0,13 & -2,66\end{array}$

encontradas se relacionan con variaciones típicas en tiempo y espacio propias de cada una de las poblaciones de esta especie.

Acorde a Vargas (1998) y Andrade (2003), se establece una relación directa entre la longevidad y la longitud máxima $\left(\mathrm{L}_{\infty}\right)$, y una relación inversa entre estas y la curvatura $(\mathrm{K})$ obtenida a partir de la función de crecimiento; indudablemente esto genera variaciones de orden matemático cuando se presentan los resultados por los diversos autores (Cuadro 2). De igual forma la obtención de la edad en función de la lectura de otolitos (Fig. 5) implica la consideración de un crecimiento proporcional, excepto

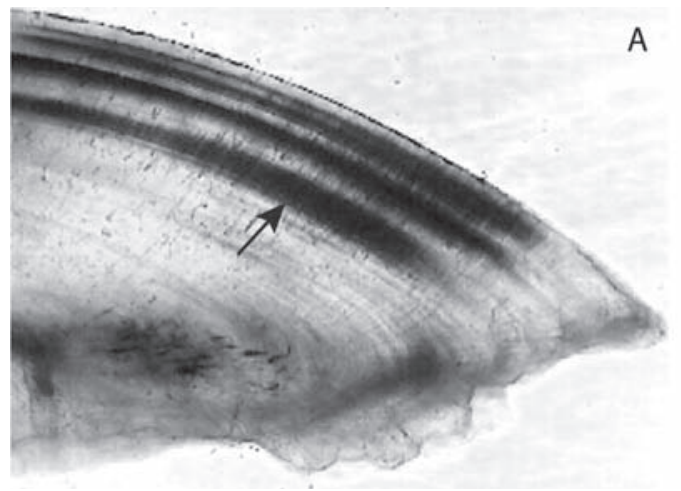

por las variaciones ambientales o de estrés donde el crecimiento somático se vea inhibido o alterado, produciendo divergencias en la determinación de la edad para ciertos especímenes dentro de la misma población (Araya y Cubillos 2002). Sin embargo, la generalidad de la determinación de edad sobre una cohorte y en general sobre una población a lo largo del tiempo en una zona tropical a partir de la lectura de otolitos se encuentra confirmada como adecuada por Newman et al. (1996), Cappo et al. (2000) y Kamukuru et al. (2005).

Así mismo, deben considerarse las variaciones que puede generar la presión por pesca

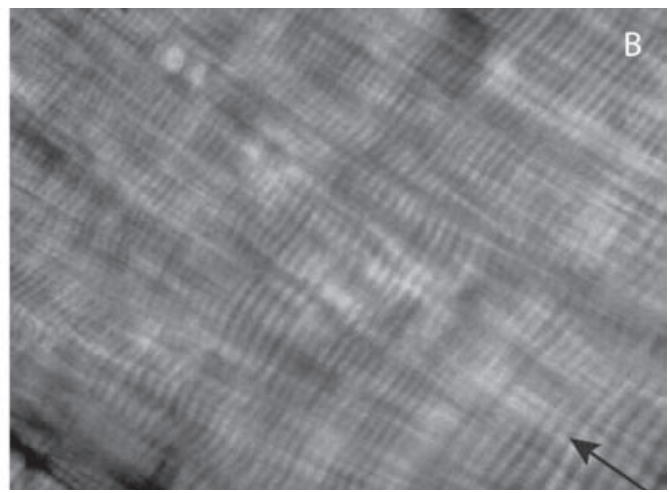

Fig. 5. Curva de crecimiento de pargo mancha en el Golfo de Nicoya, Costa Rica.

Fig. 5. Growth curve for pargo mancha in the Gulf of Nicoya, Costa Rica. 
en aquellas especies que son objeto de explotación y que por tanto deben tender a un equilibrio dinámico entre: la mortalidad por pesca, la mortalidad natural, el reclutamiento y la tasa de reproducción absoluta; $y$, darse entonces una generación de variaciones focalizadas, según se incremente o disminuya el esfuerzo en forma proporcional, dado que existe un fuerte ligamen con la distribución de edades y la abundancia poblacional, y con los cambios dependientes de la densidad tales como son la madurez y la edad de la primera madurez (Newman et al. 1996, Vargas 1998, Kamukuru et al. 2005).

La aplicación experimental cuando se obtienen las tallas de los especímenes de una especie y se realiza la determinación de la edad correspondiente, establece una clave de talla-edad apropiada (Cuadro 1); siendo posible en una forma práctica y poco costosa definir la edad con sólo medir la longitud total que poseen los organismos en estudio, y lográndose esto con una precisión bastante aceptable $\left(\mathrm{R}^{2}=0.954\right)$. Con este procedimiento se ha calibrado de previo, y con los costos propios, la metodología propia de la determinación de edad. Sin embargo, es recomendable el recalibrar la clave talla-edad cada cierta cantidad de años (3 a 5 años), conforme con las variaciones dinámicas de la población, tanto por las que sobre ella sean ejercidas como por las que se produzcan inherentes a sí misma, siendo una de las principales la presión por pesca (Anderson y Newman 1996).

Con el fin de garantizar la sostenibilidad de la pesquería a largo plazo, es recomendable establecer al menos un periodo de veda en el cual se proteja alguno de los desoves del año. Por otro lado, al relacionar la información suministrada por la clave talla- edad con la edad de primera madurez y tallas mínimas de captura, es posible establecer pautas de manejo en la pesquería de esta especie en el Golfo de Nicoya, como por ejemplo permite conocer aquellas edades que representan las mayores capturas de la especie y por tanto determinar las variaciones a mediano y largo plazo respecto a las condiciones endógenas y exógenas que afectan las tallas de captura.

\section{AGRADECIMIENTOS}

Esta investigación es parte del Proyecto Manejo Sostenible de la Pesquería en el Golfo de Nicoya, de la Universidad Nacional de Costa Rica (UNA), la Agencia Internacional de Cooperación de Japón (JICA) y el Instituto Costarricense de Pesca y Acuacultura (INCOPESCA). Se agradece a Ryo Kimura y a Satoshi Katayama, ambos del NFRS de Japón, por su colaboración en la aplicación de las técnicas para observación y análisis de otolitos.

\section{RESUMEN}

Una de las pesquerías más importantes en el Golfo de Nicoya es la del pargo mancha (Lutjanus guttatus); su captura se realiza con diversas artes y principalmente por la flota artesanal. Estudiamos especímenes de la captura comercial artesanal entre el 2002 y 2006. Se analizaron las relaciones morfométricas para aplicar la ecuación $\mathrm{P}=\mathrm{aL}^{\mathrm{b}}$; el análisis del estado de madurez gonadal se hizo macroscópicamente; se determinó la edad a través de la lectura de anillos anuales de los otolitos sagitales y los parámetros de crecimiento fueron aplicados en la ecuación de von Bertalanffy: $\mathrm{L}(\mathrm{t})=\mathrm{L}_{\infty} *\left[1-\exp \left(-\mathrm{K}^{*}\left(\mathrm{t}-\mathrm{t}_{0}\right)\right)\right]$. La relación longitud total-peso total es $\mathrm{y}=0.0236 \mathrm{x}{ }^{2.8153} \mathrm{y}$ longitud total-peso eviscerado $\mathrm{y}=0.0216 \mathrm{x}^{2.8129}$; las relaciones simplificadas correspondientes fueron $\mathrm{y}=0.0173 \mathrm{x}^{3} \mathrm{y} \mathrm{y}=$ $0.0162 \mathrm{x}^{3}$. La reproducción es todo el año con dos picos en marzo y setiembre (época seca y lluviosa, respectivamente). La proporción macho-hembra es 1:1. Se generó para la población del Golfo de Nicoya una clave edad-talla donde su utilidad se presenta como adecuada hasta los 6 años de edad. La curva de crecimiento von Bertalanffy es L $(t)=65,9\left(1-e^{-0,13(t+2,66)}\right)$.

\section{REFERENCIAS}

Anderson, R. \& R. Newman. 1996. Length, weight and associated structural indices, p. 447-482. In: B. Murphy \& D. Willis (eds.). Fisheries Techniques. Maryland, EEUU.

Andrade, H. 2003. Age determination in the snapper Lutjanus guttatus (Pisces, Lutjanidae) and investigation of fishery management strategies in the Pacific Coast of Guatemala. Master Science thesis, Norwegian College of Fisheries Science, University of Tromsø, Tromsø, Noruega.

Araya, H., A. Vásquez, B. Marín, J. A. Palacios, R. Soto Rojas, F. Mejía-Arana, Y. Shimazu \& K. Hiramatsu. 2007. Reporte no.1/2007 del Comité de Evaluación 
de Poblaciones: Reporte del Manejo de los Recursos Pesqueros en el Golfo de Nicoya. In: Proyecto Manejo Sostenible de la Pesquería en el Golfo de Nicoya: Presentación de conclusiones y recomendaciones. UNA-JICA-INCOPESCA. Puntarenas, Costa Rica.

Araya, M. \& L. Cubillos. 2002. El análisis retrospectivo del crecimiento en peces y sus problemas asociados. Gayana 66: 161-179.

Arellano-Martínez, M., A. Rojas-Herrera, F. GarcíaDomínguez, B. Ceballos-Vásquez \& M. VillalejoFuerte. 2001. Ciclo reproductivo del pargo lunarejo Lutjanus guttatus (Steindachner, 1869) en las costas de Guerrero, México. Rev. Biol. Mar. Oceanogr. 36: $1-8$.

Bussing, W. \& M. López. 1994. Peces demersales y pelágicos del pacífico de Centroamérica meridional, Rev. Biol. Trop., Universidad de Costa Rica, Vol. 1. Universidad de Costa Rica, San José, Costa Rica.

Cappo, M., P. Eden, S. Newman \& S. Robertson. 2000. A new approach to validation of periodicity and timing of opaque zone formation in the otoliths of eleven species of Lutjanus from the central Great Barrier Reef. Fish B-NOAA 98: 474-488.

Claro, R. 1994. Ecología de los peces marinos de Cuba. Instituto de Oceanología, Academia de Ciencias de Cuba y Centro de Investigaciones de Quintana Roo. Quintana Roo, México.

Cruz-Romero, M., E.A. Chávez, E. Espino \& A. García. 1996. Assessment of a snapper complex (Lutjanus spp.) of the eastern tropical Pacific. En: F. ArreguinSanchez, J.L. Munro, M.C. Balgos \& D. Pauly (eds.) Biology, fisheries and culture of tropical groupers and snappers. ICLARM Conf. Proc. No. 48, Manila, Filipinas

Fisher, W., F. Krupp, W. Schneider, C. Sommer, K.E. Carpenter \& V.H. Niem. 1995. Guía FAO para la identificación de especies para los fines de la pesca: Pacifico Centro-Oriental. Vol. III. Roma, Italia.

Kamukuru, A., T. Hetcht \& Y. Mgaya. 2005. Effects of exploitation on age, growth and mortality of the black spot snapper, Lutjanus fulviflamma, at Mafia Island, Tanzania. Fisheries Manag. Ecol. 12: 45-55.

Kimura, R. 2003. Otolith annual ring preparation manual for pargo mancha (Lutjanus guttatus) in Costa Rica. [Manuscript]. UNA-JICA-INCOPESCA, Universidad Nacional. Puntarenas, Costa Rica.

King, M. 1995. Fisheries biology: Assessment and Management. Oxford, Oxford, Londres, Inglaterra.
Márcano, L., J. Alió \& D. Altuve. 2002. Biometría y talla de primera madurez de la Tonquilla, Cynoscion jamaicensis, de la Costa Norte de la Península de Paria, Estado Sucre, Venezuela. Rev. Zootécnica Tropical. 20: 89-103.

Newman, S.J., D.McB. Williams \& G.R. Russ. 1996. Age validation, growth and mortality rates of the tropical snappers (Pisces: Lutjanidae) Lutjanus adetii (Castelnau, 1873) and L. quinquelinaeatus (Bloch, 1790) from the Central Great Barrier Reef, Australia. Mar. Freshwater Res. 47: 575-584.

Pollar, D. 1972. The biology of a landlocked from the normally catadromous salmoni form fish Galaxias maculates (JENYNS) 3. Structure of the gonads. Aust. J. Mar. Freshwater. Res. 23: 17.

Robertson, D.R. \& G.R. Allen. 2002. Shorefishes of the tropical eastern Pacific: an information system. Smithsonian Tropical Research Institute. Balboa, Panamá.

Rojas, R. 1997. Fecundidad y épocas de reproducción del pargo mancha Lutjanus guttatus (Pisces: Lutjanidae) en el Golfo de Nicoya, Costa Rica. Rev. Biol. Trop. 44: 477-487.

Siefke, K. 1995. Zur Fischerei und Populations dynamik des "pargo de la mancha" (Lutjanus guttatus) im Golf von Nicoya, Costa Rica. Tesis de Maestría. Centro de Ecología Marina Tropical. Universidad de Bremen, Bremen, Alemania.

Shimose, T \& K. Tachihara. 2005. Age, growth and maturation of the black spot snapper Lutjanus fulviflammus around Okinawa Island, Japan. Fisheries Sci. 71: 48-55.

Sparre, P. \& S. Venema. 1995. Introducción a la evaluación de los recursos pesqueros tropicales, Parte 1-Manual. FAO, Roma, Italia.

Tanaka, S. 1985. Suisan Shigengaku Soron (in Japanese). Koseisha Koseikaku, Tokio, Japón.

Vargas, M. 1998. Estimación de parámetros biológicopesqueros para el pargo mancha Lutjanus guttatus en el Golfo de Nicoya, Costa Rica. Uniciencia 15-16: 79-84.

\section{REFERENCIA DE INTERNET}

Froese, R. \& D. Pauly. 2007. Fishbase on line. www.fishbase.org (Diciembre 2007) 
